\title{
Post-intensive Care Syndrome
}

\author{
Muhammad Hanif Ahmad, Shyh Poh Teo \\ Geriatrics and Palliative Unit, Department of Internal Medicine, Raja Isteri Pengiran Anak Saleha (RIPAS) Hospital, Brunei Darussalam
}

Corresponding Author:

Shyh Poh Teo, MBChB, FRACP

Geriatrics and Palliative Unit,

Department of Internal Medicine, Raja

Isteri Pengiran Anak Saleha (RIPAS)

Hospital, Jalan Putera Al-Muhtadee

Billah, Bandar Seri Begawan, BA1710,

Brunei Darussalam

E-mail: shyhpoh.teo@moh.gov.bn

ORCID:

https://orcid.org/0000-0002-6117-5774

Received: May 10, 2021

Revised: May 31, 2021

Accepted: June 11, 2021
The increasing survival rate after discharge from the intensive care unit (ICU) has revealed longterm impairments in the cognitive, psychiatric, and physical domains among survivors. However, clinicians often fail to recognize this post-ICU syndrome (PICS) and its debilitating effects on family members (PICS-F). This study describes two cases of PICS to illustrate the different impairments that may occur in ICU survivors. The PICS risk factors for each domain and the interactions among risk factors are also described. In terms of diagnostic evaluation, limited evidence-based or validated tools are available to assist with screening for PICS. Clinicians should be aware to monitor for its symptoms on the basis of cognitive, psychiatric, and physical domains. The Montreal Cognitive Assessment is recommended to screen for cognition, as it has a high sensitivity and can evaluate executive function. Mood disorders should also be screened. For mobile patients, a 6-minute walk test should be performed. PICS can be prevented by applying the ABCDEF bundle $A B C D E F$ bundle in ICU described in this paper. Finally, the family members of patients in the ICU should be involved in patient care and a tactful communication approach is required to reduce the risk of PICS-F.

Key Words: Critical care, Neurocognitive disorders, Patient care bundles, Continuity of patient care, Physical and rehabilitation medicine

\section{INTRODUCTION}

\section{Case 1}

Mr. A, a 74-year-old man, presented to the emergency department (ED) with a fever and rigor 2 days after an ultrasound-guided transrectal prostate biopsy. He had a history of benign prostate hypertrophy, stage 2 chronic renal failure, hypertension, and hyperlipidemia and was an ex-smoker of 60 pack-years. He was hypotensive and had generalized tonic-clonic seizures at arrival. He was admitted to the intensive care unit (ICU) for septic shock and was treated with intravenous broad-spectrum antibiotics, inotropic support, and anti-epileptic drugs. His ICU admission was complicated by worsening type 1 respiratory failure due to hospital-acquired pneumonia (HAP). Recurrent failed extubation resulted in a tracheostomy 2 weeks later and 1 month after intubation, before he was successfully weaned off ventilatory support. He was transferred out of the ICU after 5 weeks to a medical ward fully dependent on nasogastric feeding. The geriatric medical team engaged allied health professionals for his care, including physiotherapy, occupational therapy, dietitian, and speech-language therapy. Despite fatigue initially limiting his participation in rehabilitation and complications from HAP and upper gastrointestinal bleeding, he gradually showed improvement under the individualized graded exercise program. On discharge after 4 months in hospital, the patient was able to mobilise with a zimmer frame. Within 1 year, tracheostomy decannulation was performed and the patient returned to normal oral feeding, and in 18 months, the patient fully recovered to independence in all activities of daily living (ADLs).

Three years later, the patient presented to the Geriatrics Clinic for auditory and visual hallucinations, insomnia, and nocturnal wandering. He self-reported cognitive decline, particularly shortterm memory loss and word-finding difficulty. His family noted 
increasing agitation and irritability, as well as increasing difficulties using a cellphone and performing prayers. He denied having depression or anxiety. His wife complained of increased stress because of his behavioral changes, in addition to sleep deprivation and disruption of her daily routine. His Montreal Cognitive Assessment (MoCA) score was 7/20, with memory deficits, delayed recall, language, abstraction, naming, visuospatial, and executive function. He was diagnosed as having moderate-to-severe dementia with behavioral and psychological symptoms of dementia and post-intensive care syndrome (PICS).

\section{Case 2}

Ms. T, a 70-year-old woman, presented to the ED with a 4-day history of fever and cough. She had a history of hypertension, hyperlipidemia, and uncomplicated type 2 diabetes mellitus. She was hypoxic and hypotensive despite initial fluid resuscitation and non-invasive ventilatory support. She was admitted to the ICU for severe community-acquired pneumonia, rapid atrial fibrillation, and multi-organ failure. She required short-term hemodialysis for renal support and prolonged intravenous antibiotics for ongoing bacteremia. She underwent a tracheostomy owing to her prolonged need for ventilatory support and nasogastric feeding. After 2 months in the ICU, she was transferred to a geriatric medicine ward and was fully dependent for all ADLs. Her rehabilitation was complicated by fatigue, anxiety, and labile glycemic control. She was discharged after 3 months of hospitalization and was able to transfer to a wheelchair with assistance.

Tracheostomy decannulation, normal oral diet, and return to independent mobility were achieved within 1 year. During the clinical follow-up, she reported having anxiety, with occasional flashbacks of her ICU admission. Three years later, she reported having short-term memory loss, which did not affect her ADLs. The patient was diagnosed as having mild cognitive impairment and PICS.

\section{Background}

Although survival rates in patients with critical illness and requiring ICU care are increasing, survivors may develop long-term impairments within one or more of the cognitive, psychiatric, and physical domains. ${ }^{1)}$ This cluster of impairments is known as PICS. This diagnosis excludes patients having traumatic brain injuries or stroke. Similar debilitating effects on family members, particularly psychological symptoms among those caring for the patient in the ICU and after discharge, are termed post-intensive care syndrome-family (PICS-F).

It is important to be aware of this condition in older patients. The number of older people with critical illnesses is increasing with an increase in the aging population. Compared with other patient populations, older patients account for more ICU admissions and have a high risk of delirium, which is a risk factor for developing cognitive dysfunction in PICS. ${ }^{2,3)}$

Approximately 50\%-70\% of all ICU survivors have at least one PICS-related impairment, which can persist for up to 15 years after discharge. ${ }^{4)}$ A study of Medicare beneficiaries (mean age of 76.9 years) found that $60 \%$ of 1,520 ICU hospitalizations with severe sepsis were associated with worsening cognitive or physical functioning 1 year after hospitalization, with $16.7 \%$ of survivors showing moderate-to-severe cognitive impairment. ${ }^{5,6)}$ A retrospective cohort study of 21,520 Medicare patients revealed that ICU stays of 3 days or longer, sepsis severity, older age, frailty, depression, and dementia increased the odds of physical disability in older adults receiving home care. ${ }^{7)}$ Another study reported that $15 \%$ of older ICU survivors had a new diagnosis of dementia 3 years post-ICU stay, with almost $40 \%$ of new dementia cases diagnosed during the first year post-ICU stay.

\section{RISK FACTORS FOR PICS}

The risk factors for PICS partly depend on which among the cognitive, psychiatric, and physical domains are most affected. These three components are interrelated, with impairment in one domain frequently being associated with worsening function in another. The risk factors for each domain are summarized in Fig. 1.

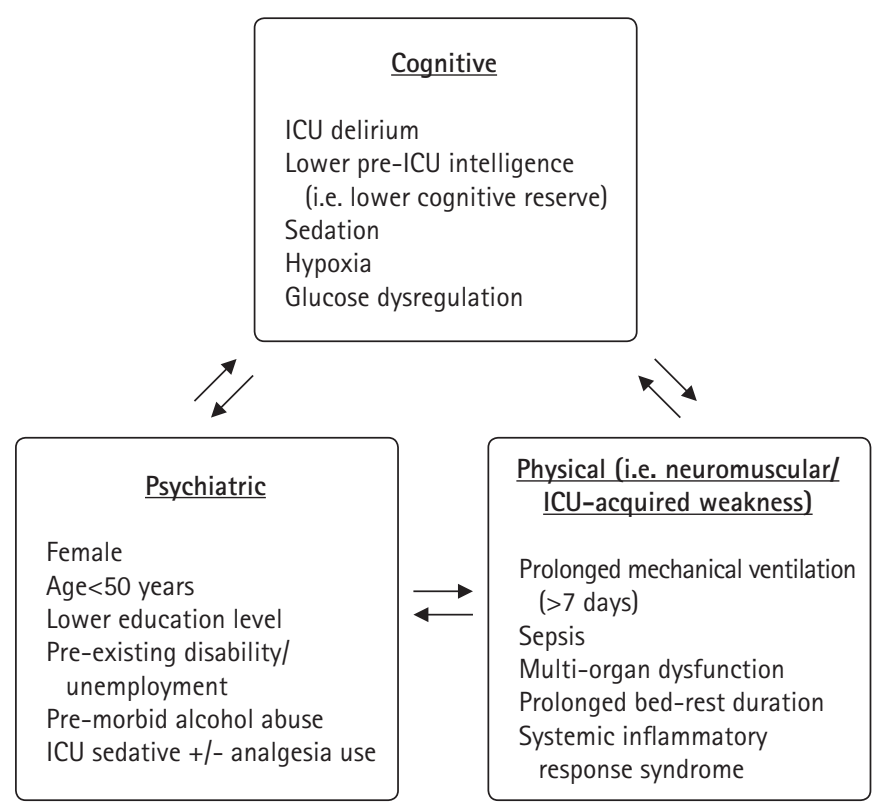

Fig. 1. The post-intensive care syndrome (PICS) risk factors for each domain. ICU, intensive care unit. 


\section{Cognitive PICS (Case 1)}

The major risk factors for cognitive PICS include delirium, poor cognitive reserve, sepsis, and acute respiratory distress syndrome. The duration of delirium is reportedly an independent risk factor for cognitive impairment at 6 and 12 months. ${ }^{9)}$ However, there is no strong evidence suggesting that older adults are at a higher risk for cognitive PICS than are younger adults. A prospective study of 821 ICU patients showed similar cognitive and executive function scores between patients aged 65 years and older and younger patients at 3 and 12 months after discharge. ${ }^{2)}$ Patients with a higher level of education, an indicator of cognitive reserve, had a lower risk of this complication at 3 and 12 months. ${ }^{10)}$ Severe sepsis survivors were three times more likely to develop moderate-to-severe cognitive dysfunction. It is unclear whether cognitive dysfunction is due to acute respiratory distress syndrome itself, complications from sepsis, or the use of mechanical ventilation.

The main cognitive functions affected include attention or concentration, mental processing speed, memory, and executive function. The latter two are necessary for engaging in purposeful, goal-directed behaviors. Loss of these functions tends to impair rehabilitation participation, resulting in poor functional outcomes. Survivors have self-reported severe cognitive impairment after ICU discharge that has persisted for up to 2 years. ${ }^{11)}$

\section{Physical PICS (Cases 1 and 2)}

Approximately 50\% of older ICU survivors show functional recovery after critical illness, with a median recovery time of 3 months. ${ }^{6}$ ) Older survivors of severe sepsis developed 1.5 new limitations in ADLs, compared with 0.5 in their non-ICU counterparts. ${ }^{12)}$ Older survivors who were mechanically ventilated were $30 \%$ more likely to have an $\mathrm{ADL}$ disability. ${ }^{13)}$ The physical impairments result in a need for increased support after hospital discharge.

Pre-existing functional disability, cognitive impairment, and frailty are strongly associated with physical PICS or ICU-acquired weakness (ICU-AW). Prolonged mechanical ventilation ( $>7$ days), immobility, ongoing inflammatory response syndrome from sepsis, and multi-organ dysfunction are also associated with the development of ICU-AW. The pathophysiology of this condition is as follows: microvascular ischemia, catabolism, and prolonged immobility cause skeletal muscle wasting, whereas microvascular injury results in nerve ischemia, nerve-related sodium channel dysfunction, and mitochondrial injury. This leads to critical illness-related neuropathy, myopathy, or both. ${ }^{14)}$

The BRAIN-ICU study found that physical disability significantly contributed to poor mental health and quality of life at 3 and 12 months post discharge. ${ }^{15)}$ However, the nature of this interaction among physical impairment, mental health, and quality of life in ICU survivors requires further evaluation.

\section{Psychiatric PICS (Case 2)}

Patients who develop critical illnesses have higher incidences of premorbid psychiatric illness than do patients who do not require ICU hospitalization and the general population. ${ }^{16)}$ Whereas ICU survivors have a 4- to 6-fold higher rate of psychiatric comorbidities than in the general population, those with psychiatric PICS tend to have underlying pre-existing psychiatric disorders. Depression, anxiety, and post-traumatic stress disorder (PTSD) are the three common manifestations of psychiatric PICS, occurring in $19 \%-37 \%, 32 \%-40 \%$, and $19 \%-22 \%$ of patients, respectively. ${ }^{6}$

\section{DIAGNOSTIC EVALUATION OF PICS}

Diagnosing PICS is challenging because of the failure of early recognition and lack of screening tools for these patients. Serial assessments using history, physical examination, investigations (if required), and multidisciplinary consultations between specialists and therapists are essential to systematically evaluate the PICS domains.

There are no validated cognitive screening tools for PICS. While the MoCA, Mini-Mental State Examination, and Mini-Cog are widely used, they are poor predictors of cognitive impairment at 6 months or longer after discharge. ${ }^{17)}$ As executive dysfunction is the main cognitive domain affected in PICS, the Society of Critical Care Medicine (SCCM) recommends using the MoCA, as it has a component on executive function and is sensitive for detecting mild cognitive impairment. ${ }^{18)}$

Clinicians should also screen for mood disorders, particularly anxiety, depression, and PTSD. ${ }^{19)}$ Again, there are no adequately validated questionnaires for survivors of critical illnesses. The commonly used tools include the Hospital Anxiety and Depression Scale (HADS), Impact of Events Scale-Revised (IES-R), and the six-item Impact of Event Scale-6 (IES-6). If a mood disorder is identified, patients should be asked about sexual health problems and sleep hygiene, as both issues tend to occur concurrently and may worsen psychiatric PICS.

The manifestations of physical PICS (ICU-AW) can range from generalized poor mobility and multiple falls to generalized muscular weakness. This can be due to disuse or deconditioning; however, definitive diagnoses should be sought for critical illness myopathy (CIM), critical illness polyneuropathy (CIP), and critical illness polyneuromyopathy (CIPNM). CIM tends to be more proximal than distal weakness with sensory preservation and atrophy depending on the illness duration, whereas CIP is more distal than proximal, with limited atrophy. CIPNM is a combination of proxi- 
mal and distal weaknesses, with distal sensory loss and variable atrophy. For all three types, reflexes tend to be preserved initially but gradually disappear as the weakness progresses, sparing the bulbar musculature. ${ }^{20)}$

It may be challenging to specify the diagnosis of physical PICS because of the overlap of the conditions and the limited clinical examination possible for sedated or intubated patients. For example, it may be difficult to differentiate between CIP in patients with pre-existing diabetic peripheral neuropathy before ICU admission. However, as with both patients described here, failure to wean off ventilatory support may be an early indicator of physical PICS, requiring confirmatory electrodiagnostic tests such as nerve conduction studies and electromyography.

One of the main tests used to measure changes in strength and functional outcomes in the ICU is the physical function outcome measure (PFIT). This tool was initially developed to measure endurance, strength, cardiovascular capacity, and functional level among mechanically ventilated tracheostomy patients who were able to stand, with the test repeated after weaning from ventilation. ${ }^{21)}$ The tests involved assessments of strength for shoulder flexion and knee extension, level of assistance required for sit-tostand, and step cadence in steps per minute. The PFIT demonstrated high inter-rater reliability and sensitivity in ICU patients. ${ }^{22}$

In the ICU setting, the 6-minute walk test (6MWT) and Timed Up and Go (TUG) test may not be practical, as they require space and consideration of how to manage drips, drains, and oxygen delivery systems when the patient is walking or turning. However, a 6MWT should be performed in extubated and mobile ICU patients. A randomized controlled trial of patients admitted to the ICU for 5 days or longer comparing the results of the $6 \mathrm{MWT}$, TUG, and PFIT at admission, discharge, and follow-up showed that the $6 \mathrm{MWT}$ was able to demonstrate the rate of change over time and between-group differences for the intervention group (intensive exercises in ICU, wards, and outpatients) and the control group (standard care). ${ }^{23)}$ Thus, for ICU patients, although the PFIT may be used for initial assessment, the 6MWT should be used for follow-up once the patient is extubated and able to start mobilizing.

It is important to obtain early input from physiotherapists and occupational therapists for assessment and management, including preemptive prevention of complications such as contractures, preferably while the patient is still in the ICU. ${ }^{20,24)}$

Comprehensive geriatric assessment should be performed, with continual assessment and rehabilitation until discharge. Dietitian input for nutrition assessment and intervention is important to ensure that nutritional requirements are met during the recovery phase.
It is also crucial to identify the presence and severity of pre-existing illnesses within the three domains, as unchanged symptoms do not support the diagnosis of PICS. This is challenging for those without the cognitive capacity to compare their current status with symptoms before admission. Engagement with close family members is beneficial in identifying the presence and nature of pre-existing comorbidities. In addition to collateral history obtained from caregivers and family, pre-existing clinical records of diagnoses, investigations, cognitive tests, and functional assessments, such as from previous physiotherapy sessions (if available), are valuable in objectively confirming acute changes post-ICU stay compared with baseline. Unfortunately, the cognitive status before ICU admission, particularly objective measures, is usually unavailable. A systematic review also found that most publications on PICS lacked information on baseline cognitive status; thus, it was not possible to determine whether the observed cognitive deficits arose de novo or represented worsening of pre-existing cognitive deficits. $^{25)}$

\section{MANAGEMENT OF PICS}

The management of PICS is divided into two parts: within the ICU and post-ICU stay. Intensivists can reduce the risk of PICS by minimizing sedation, avoiding psychotropic use including antipsychotics and anticholinergic agents, avoiding hypoglycemia and hypoxemia, environmental modifications, and prioritizing early physical rehabilitation and mobility for older people. The ABCDEF bundle approach shown in Fig. 2 has been shown to reduce the likelihood of death within 7 days, mechanical ventilation, coma, delirium, and the use of physical restraints. ${ }^{26,27)}$

Treatment of patients with PICS involves managing individual impairments in each domain through multidisciplinary care. The physician responsible should coordinate care with other clinicians and allied health professionals. Early physical therapy once a patient is stable from cardiorespiratory and neurological perspectives may decrease cognitive impairment, improve physical function, and reduce psychiatric comorbidity. Cognitive enhancers and cognitive rehabilitation through memory training are not effective in PICS. Thus, further studies are needed to identify evidence-based treatments for this condition.

Although there is limited evidence to guide the discontinuation of antipsychotics in the post-ICU phase, it is recommended to use the smallest dose possible for behavioral disturbance from postoperative delirium and to discontinue their administration as soon as possible. ${ }^{16)}$ If antipsychotics are required after discharge, the benefits versus risks should be considered for individual patients.

The optimal follow-up and natural progression of PICS remain 


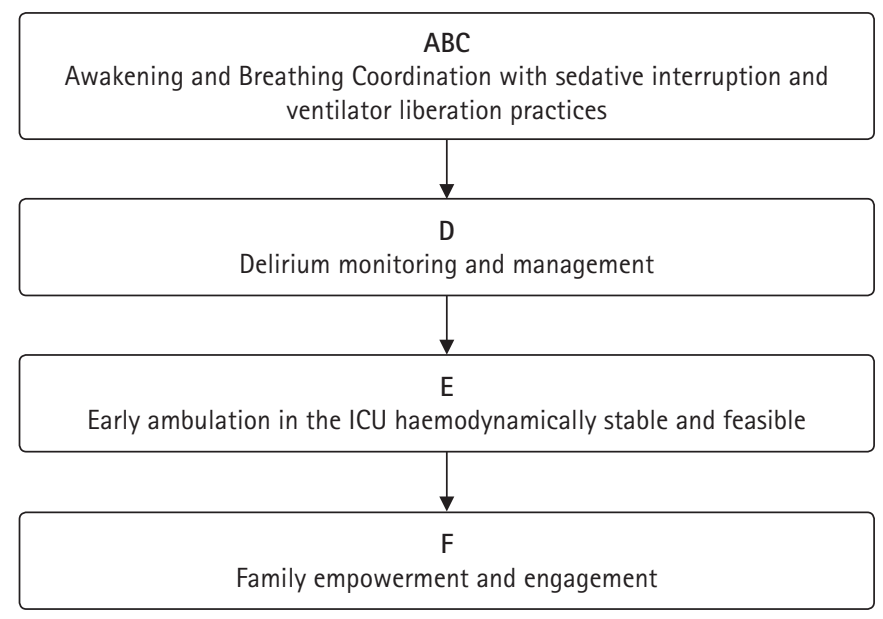

Fig. 2. The ABCDEF bundle approach. ICU, intensive care unit.

uncertain, but most patients are followed up for several years at regular intervals. We recommend follow-ups every 3 months after discharge, followed by individualized intervals according to the needs of each patient. This is important, as symptoms may become more apparent at a later stage, as seen in Case 1. The care of older people with PICS requires a multidisciplinary approach, including intervention by medical specialists, general practitioners, therapists, and social services. If available, referrals to established PICS peer support groups may assist in reducing psychological stress. Although PICS clinics have been introduced in the UK and the United States, evidence of their benefits remains conflicting.

The symptoms and signs of PICS improve modestly over the first 6 to 12 months after ICU discharge. However, most patients have deficits persisting for years, with an associated inability to work, a reduced quality of life, and an increased risk of mortality. ${ }^{28)}$ The effects of preventative or therapeutic interventions on these outcomes remain unknown, as is the risk for subsequent hospitalizations, critical illnesses, or PICS recurrence.

An important component of the $\mathrm{ABCDEF}$ bundle is family empowerment and engagement. Clinicians should evaluate family members for PICS-F, as they may also develop persistent physical and psychological symptoms after patient discharge. The risk factors for PICS-F are divided into those related to caregivers, patients, and the healthcare system. Caregiver factors associated with the development of PICS-F include female sex, spouse caregiver, low education level, pre-existing mental or physical illness, family history of mental illness, lack of social and professional support, and caregiving for more than 100 hours per month. ${ }^{29)}$ Healthcare-system or ICU-related factors are potentially modifiable, such as limited ICU visiting hours, patient perception of being near death, and communication skills of ICU physicians. Patient-related factors play only a small role in the development of PICS-F, ac- cording to the findings of the RECOVER study. ${ }^{30)}$

The SCCM recommends family involvement in the care of ICU patients. However, these guidelines lack recommendations on how and when to screen family members and who is responsible for assessing and managing PICS-F. Although further research is required to validate screening tools for PICS-F, we recommend close monitoring of family member well-being and considering referral for psychotherapy if indicated.

Studies have shown that the following approaches are useful to improve both patient and family outcomes, resulting in a high level of family satisfaction and reduced family anxiety: creation of "open" ICUs, family witness of resuscitation efforts, ICU diaries, and proactive engagement of family members in patient care. ${ }^{31,32)}$ "Open" ICUs are characterized by flexible visiting policies in terms of hours, number or age of visitors, daily meetings with family members, healthcare providers performing tasks in front of family members, and redesigning units to ensure family comfort and sleep needs. Incorporating family members into ICU care and witnessing codes have been shown to reduce depression and PTSD risk at 3 and 12 months post discharge. ${ }^{33)}$ Most studies support the use of ICU diaries to document events chronologically, with entries from staff and family members and photographs of patients. ${ }^{34)}$

Effective, structured communication strategies between staff and family members are also useful in reducing the risk of developing PICS-F. Useful approaches include conducting a family conference within 72 hours of ICU admission, ensuring consistent communication from different team members, and increasing the proportion of time spent listening rather than talking. Empathy is required to recognize the difficulties of being a surrogate decision-maker and having a critically ill or experiencing the impending loss of a loved one. Clinicians should identify opportunities to acknowledge and address family emotions, explore patient values and treatment preferences, explain the role of surrogate decision-makers, and affirm non-abandonment of the patient and family, even if the patient is dying. ${ }^{35)}$ These principles are summarized in the mnemonic "VALUE" (Value family statements, Acknowledge and Listen to family emotions, Understand patients as persons, and Elicit family questions) and have been shown to reduce PICS-F symptoms and increase family confidence in the care team. ${ }^{36,37)}$

Evidence is limited or lacking regarding specific post-ICU interventions to reduce symptoms of PICS or PICS-F. ${ }^{38)}$ A major barrier to conducting large-scale, multi-site studies is the variability of post-ICU follow-up. Patients and caregivers, particularly in rural and underserved areas, may receive follow-up at different hospitals or healthcare systems from their initial ICU admission. In addition to rehabilitation and follow-up, we recommend that patients and 
caregivers be provided access to support services such as home health care and the integration of care with the involvement of primary care physicians before discharge.

Whereas post-ICU clinics can provide interventions for the management of PICS-F, family-centered care remains inconsistent, with ambiguity in which healthcare professionals are responsible for managing PICS-F. This is a significant issue, as the effects of PICS-F may be long-lasting and profoundly impact caregivers' quality of life.

Meanwhile, clinical practice guidelines and protocols are needed for the diagnosis and management of the physical and neuropsychiatric sequelae of PICS. These interventions should be system-based yet individualized to each ICU survivor and developed through collaboration among intensivists, geriatricians, and multidisciplinary teams. A recovery care coordinator should also be designated to enable care coordination for the patient and caregivers from the ICU, as well as the implementation of individual care plans to facilitate a smooth transition of care from ICU to outpatient settings. ${ }^{2)}$

Once there is improved recognition and identification of PICS, further research is needed to create an evidence-based approach and model of care to effectively manage this condition. More research is also required to elucidate the mechanism for each component of PICS (cognitive, physical, and psychiatric), develop sensitive screening tools and specific confirmatory tests, and evaluate the effectiveness of preventive and treatment approaches for this condition.

\section{CONCLUSION}

There is limited awareness and under-recognition of PICS among clinicians despite its significant impact on the function and quality of life of patients and families. This condition may be prevented by encouraging early mobility in the ICU and minimizing the use of sedatives and antipsychotics. Once PICS is identified, rehabilitation, management, and follow-up should be carried out by a multidisciplinary team. Further research is required to strengthen the evidence base for the diagnosis and management of PICS and PICS-F.

\section{ACKNOWLEDGMENTS}

\section{CONFLICT OF INTEREST}

The researchers claim no conflicts of interest.

\section{AUTHOR CONTRIBUTION}

Conceptualization, MHA, SPT; Data curation, MHA, SPT; Methodology, MHA, SPT; Project administration, MHA, SPT; Supervision, SPT; Writing-original draft, MHA, SPT; Writing-review \& editing, MHA, SPT.

\section{REFERENCES}

1. Iwashyna TJ. Survivorship will be the defining challenge of critical care in the 21st century. Ann Intern Med 2010;153:204-5.

2. Wang S, Allen D, Kheir YN, Campbell N, Khan B. Aging and post-intensive care syndrome: a critical need for geriatric psychiatry. Am J Geriatr Psychiatry 2018;26:212-21.

3. Baldwin MR. Measuring and predicting long-term outcomes in older survivors of critical illness. Minerva Anestesiol 2015;81: 650-61.

4. Desai SV, Law TJ, Needham DM. Long-term complications of critical care. Crit Care Med 2011;39:371-9.

5. Iwashyna TJ, Ely EW, Smith DM, Langa KM. Long-term cognitive impairment and functional disability among survivors of severe sepsis. JAMA 2010;304:1787-94.

6. Ferrante LE, Pisani MA, Murphy TE, Gahbauer EA, Leo-Summers LS, Gill TM. Factors associated with functional recovery among older intensive care unit survivors. Am J Respir Crit Care Med 2016;194:299-307.

7. Riegel B, Huang L, Mikkelsen ME, Kutney-Lee A, Hanlon AL, Murtaugh CM, et al. Early Post-intensive care syndrome among older adult sepsis survivors receiving home care. J Am Geriatr Soc 2019;67:520-6.

8. Guerra C, Hua M, Wunsch H. Risk of a diagnosis of dementia for elderly Medicare beneficiaries after intensive care. Anesthesiology 2015;123:1105-12.

9. Pandharipande PP, Girard TD, Jackson JC, Morandi A, Thompson JL, Pun BT, et al. Long-term cognitive impairment after critical illness. N Engl J Med 2013;369:1306-16.

10. Marra A, Pandharipande PP, Girard TD, Patel MB, Hughes CG, Jackson JC, et al. Co-occurrence of post-intensive care syndrome problems among 406 survivors of critical illness. Crit Care Med 2018;46:1393-401.

11. Mikkelsen ME, Christie JD, Lanken PN, Biester RC, Thompson BT, Bellamy SL, et al. The adult respiratory distress syndrome cognitive outcomes study: long-term neuropsychological function in survivors of acute lung injury. Am J Respir Crit Care Med 2012;185:1307-15.

12. Iwashyna TJ, Cooke CR, Wunsch H, Kahn JM. Population burden of long-term survivorship after severe sepsis in older Americans. J Am Geriatr Soc 2012;60:1070-7.

\section{FUNDING}

None. 
13. Barnato AE, Albert SM, Angus DC, Lave JR, Degenholtz HB. Disability among elderly survivors of mechanical ventilation. Am J Respir Crit Care Med 2011;183:1037-42.

14. Fan E, Dowdy DW, Colantuoni E, Mendez-Tellez PA, Sevransky JE, Shanholtz C, et al. Physical complications in acute lung injury survivors: a two-year longitudinal prospective study. Crit Care Med 2014;42:849-59.

15. Jackson JC, Pandharipande PP, Girard TD, Brummel NE, Thompson JL, Hughes CG, et al. Depression, post-traumatic stress disorder, and functional disability in survivors of critical illness in the BRAIN-ICU study: a longitudinal cohort study. Lancet Respir Med 2014;2:369-79.

16. Wunsch H, Christiansen CF, Johansen MB, Olsen M, Ali N, Angus DC, et al. Psychiatric diagnoses and psychoactive medication use among nonsurgical critically ill patients receiving mechanical ventilation. JAMA 2014;311:1133-42.

17. Woon FL, Dunn CB, Hopkins RO. Predicting cognitive sequelae in survivors of critical illness with cognitive screening tests. Am J Respir Crit Care Med 2012;186:333-40.

18. Mikkelsen ME, Still M, Anderson BJ, Bienvenu OJ, Brodsky MB, Brummel N, et al. Society of Critical Care Medicine's International Consensus Conference on prediction and identification of long-term impairments after critical illness. Crit Care Med 2020; 48:1670-9.

19. Wolters AE, Peelen LM, Welling MC, Kok L, de Lange DW, Cremer OL, et al. Long-term mental health problems after delirium in the ICU. Crit Care Med 2016;44:1808-13.

20. Shepherd S, Batra A, Lerner DP. Review of critical illness myopathy and neuropathy. Neurohospitalist 2017;7:41-8.

21. Skinner EH, Berney S, Warrillow S, Denehy L. Development of a physical function outcome measure (PFIT) and a pilot exercise training protocol for use in intensive care. Crit Care Resusc 2009; 11:110-5.

22. Denehy L, de Morton NA, Skinner EH, Edbrooke L, Haines K, Warrillow S, et al. A physical function test for use in the intensive care unit: validity, responsiveness, and predictive utility of the physical function ICU test (scored). Phys Ther 2013;93:163645.

23. Denehy L, Skinner EH, Edbrooke L, Haines K, Warrillow S, Hawthorne G, et al. Exercise rehabilitation for patients with critical illness: a randomized controlled trial with 12 months of follow-up. Crit Care 2013;17:R156.

24. Fan E, Cheek F, Chlan L, Gosselink R, Hart N, Herridge MS, et al. An official American Thoracic Society Clinical Practice guideline: the diagnosis of intensive care unit-acquired weakness in adults. Am J Respir Crit Care Med 2014;190:1437-46.

25. Kohler J, Borchers F, Endres M, Weiss B, Spies C, Emmrich JV.
Cognitive deficits following intensive care. Dtsch Arztebl Int 2019;116:627-34.

26. Morandi A, Brummel NE, Ely EW. Sedation, delirium and mechanical ventilation: the 'ABCDE' approach. Curr Opin Crit Care 2011;17:43-9.

27. Pun BT, Balas MC, Barnes-Daly MA, Thompson JL, Aldrich JM, Barr J, et al. Caring for critically ill patients with the ABCDEF bundle: results of the ICU Liberation Collaborative in over 15,000 adults. Crit Care Med 2019;47:3-14.

28. Rydingsward JE, Horkan CM, Mogensen KM, Quraishi SA, Amrein K, Christopher KB. Functional status in ICU survivors and out of hospital outcomes: a cohort study. Crit Care Med 2016;44:869-79.

29. Davidson JE, Jones C, Bienvenu OJ. Family response to critical illness: postintensive care syndrome-family. Crit Care Med 2012;40:618-24.

30. Cameron JI, Chu LM, Matte A, Tomlinson G, Chan L, Thomas $\mathrm{C}$, et al. One-year outcomes in caregivers of critically ill patients. N Engl J Med 2016;374:1831-41.

31. Davidson JE, Powers K, Hedayat KM, Tieszen M, Kon AA, Shepard E, et al. Clinical practice guidelines for support of the family in the patient-centered intensive care unit: American College of Critical Care Medicine Task Force 2004-2005. Crit Care Med 2007;35:605-22.

32. Au SS, Roze des Ordons A, Soo A, Guienguere S, Stelfox HT. Family participation in intensive care unit rounds: comparing family and provider perspectives. J Crit Care 2017;38:132-6.

33. Jabre P, Tazarourte K, Azoulay E, Borron SW, Belpomme V, Jacob L, et al. Offering the opportunity for family to be present during cardiopulmonary resuscitation: 1-year assessment. Intensive Care Med 2014;40:981-7.

34. Blair KT, Eccleston SD, Binder HM, McCarthy MS. Improving the patient experience by implementing an ICU diary for those at risk of post-intensive care syndrome. J Patient Exp 2017;4:4-9.

35. Selph RB, Shiang J, Engelberg R, Curtis JR, White DB. Empathy and life support decisions in intensive care units. J Gen Intern Med 2008;23:1311-7.

36. Curtis JR, White DB. Practical guidance for evidence-based ICU family conferences. Chest 2008;134:835-43.

37. Lautrette A, Darmon M, Megarbane B, Joly LM, Chevret S, Adrie C, et al. A communication strategy and brochure for relatives of patients dying in the ICU. N Engl J Med 2007;356:46978.

38. Schofield-Robinson OJ, Lewis SR, Smith AF, McPeake J, Alderson P. Follow-up services for improving long-term outcomes in intensive care unit (ICU) survivors. Cochrane Database Syst Rev 2018;11:CD012701. 